\title{
A Diagnostic Panel for Acquired Immune-Mediated Polyneuropathies Based on the Expression of IncRNAs
}

\author{
Bashdar Mahmud Hussen ${ }^{\text {, Fwad Nicknafs }}{ }^{2}$, Hazha Jamal Hidayat ${ }^{3}$, Arezou Sayad ${ }^{2}$, \\ Soudeh Ghafouri-Fard ${ }^{2 *}$ and Mohammad Taheri ${ }^{4 *}$ \\ ${ }^{1}$ Pharmacognosy Department, College of Pharmacy, Hawler Medical University, Erbil, Iraq, ${ }^{2}$ Department of Medical \\ Genetics, School of Medicine, Shahid Beheshti University of Medical Sciences, Tehran, Iran, ${ }^{3}$ Department of Biology, College \\ of Education, Salahaddin University-Erbil, Erbil, Iraq, ${ }^{4}$ Urology and Nephrology Research Center, Shahid Beheshti University \\ of Medical Sciences, Tehran, Iran
}

OPEN ACCESS

Edited by:

Sara Marinelli,

National Research Council (CNR), Italy

Reviewed by:

Janine Adele Lamb,

The University of Manchester,

United Kingdom

Amin Safa,

Complutense University of

Madrid, Spain

*Correspondence:

Soudeh Ghafouri-Fard

s.ghafourifard@sbmu.ac.ir

Mohammad Taheri

mohammad_823@yahoo.com

Specialty section:

This article was submitted to

Multiple Sclerosis and

Neuroimmunology,

a section of the journal

Frontiers in Immunology

Received: 18 December 2020

Accepted: 27 January 2021

Published: 23 February 2021

Citation:

Hussen BM, Nicknafs F, Hidayat HJ,

Sayad A, Ghafouri-Fard S and

Taheri M (2021) A Diagnostic Panel for

Acquired Immune-Mediated

Polyneuropathies Based on the

Expression of IncRNAs.

Front. Immunol. 12:643615

doi: 10.3389/fimmu.2021.643615
Long non-coding RNAs (IncRNAs) have been shown to alter immune responses, thus contributing to the pathobiology of autoimmune conditions. We investigated the expression levels of ANRIL, PICART1, MALAT1, CCAT1, CCAT2, and CCHE1 IncRNAs in acute and chronic inflammatory demyelinating polyneuropathy (AIDP and CIDP). ANRIL, PICART1, MALAT1, CCAT1, CCAT2, and CCHE1 IncRNAs were significantly downregulated in individuals with both AIDP and CIDP compared with unaffected individuals. Gender-based comparisons also verified such downregulations in both male and female subjects compared with sex-matched unaffected controls for all IncRNAs. There was no significant difference in the expression of any of the IncRNAs between cases with AIDP and cases with CIDP. While the expression levels of ANRIL and PICART1 were significantly correlated in healthy subjects $(r=0.86, p=8.5 \mathrm{E}-16)$, similar analysis in cases with AIDP and CIDP revealed no significant correlation. The most robust correlation among patients was detected between ANRIL and MALAT1 IncRNAs ( $r=0.59, p=3.52 \mathrm{E}-6)$. ANRIL, MALAT1, and PICART1 had the diagnostic power of 0.96, 0.94, and 0.92 in distinguishing between cases with CIDP and controls, respectively. A combination of all IncRNAs resulted in 0.95 diagnostic power with a sensitivity of 0.85 and specificity of 0.96 for this purpose. Diagnostic power values of these IncRNAs in differentiation between cases with AIDP and controls were 0.98 , 0.95, and 0.93, respectively. The combinatorial diagnostic power reached 0.98 for differentiation between cases with AIDP and controls. The six-IncRNA panel could differentiate combined cases with AIDP and CIDP from controls with area under the curve (AUC), sensitivity, and specificity values of $0.97,0.90$, and 0.96 , respectively. Collectively, the IncRNA panel is suggested as a sensitive and specific diagnostic panel for acquired immune-mediated polyneuropathies.

Keywords: AIDP, CIDP, IncRNA, ANRIL, MALAT1, CCAT1, CCAT2, CCHE1

\section{INTRODUCTION}

Immune-mediated polyneuropathies comprise a wide spectrum of disorders with variable subtypes including the acute monophasic form, i.e., acute inflammatory demyelinating polyneuropathy (AIDP) and the chronic, corticosteroid-responsive type, namely, chronic inflammatory demyelinating polyneuropathy (CIDP). These conditions are thought to result from 
autoimmune responses against some elements of the myelin sheath of peripheral nerves or some other proteins (1). Yet, there is no constant presence of autoantibodies in all subtypes (1). The existence of several subtypes has made the diagnosis challenging (1). Currently, the diagnosis of these conditions is based on the assessment of protein levels in the cerebrospinal fluid (CSF), nerve biopsy, and electrodiagnostic methods (2). Most diagnostic criteria for these conditions propose evidence to support the diagnosis, instead of conclusive confirmation of the diagnosis (2). Therefore, it is necessary to identify diagnostic biomarkers for routine application in the clinical setting. A large body of evidence supports the putative role of long non-coding RNAs (lncRNAs) as biomarkers for autoimmune disorders $(3,4)$. A number of lncRNAs are more promising in this field, as they regulate immune responses from different points. For instance, ANRIL expression has been shown to be regulated by nuclear factor kappa $\mathrm{B}(\mathrm{NF}-\kappa \mathrm{B})$ through

TABLE 1 | Global data of enlisted persons.

\section{Variables \\ Cases with AIDP Cases with CIDP \\ Controls}

Female/male [no. (\%)] $6(27 \%) / 16(73 \%) 11(35 \%) / 20(65 \%) 25(50 \%) / 25(50 \%)$ Age $($ mean $\pm S D, Y) \quad 49.72 \pm 14.6 \quad 50.5 \pm 15.8 \quad 44.48 \pm 2.3$ tumor necrosis factor alpha (TNF- $\alpha)$. This $\operatorname{lncRNA}$ regulates the expression of a number of proinflammatory proteins (5). PICART1 has been shown to suppress JAK2/STAT3 signaling (6). STAT3 has functional roles in the regulation of immune response through its interplay with IRF4, BATF, and ROR $\gamma \mathrm{t}$ (7). MALAT1 regulates the maturation and apoptosis of dendritic cells (DCs) and the production of proinflammatory cytokine by these cells (8). CCAT1 can control the expression of interleukin-10 (IL-10) and modulate macrophage polarization (9). CCAT2 has functional interactions with TGF- $\beta$ signaling (10), a pathway that has multiple roles in the regulation of immune responses through the regulation of thymic $\mathrm{T}$ cell selection, the preservation of balance of the naïve $\mathrm{T}$ cell population, the suppression of cytotoxic $\mathrm{T}$ and $\mathrm{T}$ helper cells differentiation, and the enhancement of the production of regulatory $\mathrm{T}$ cells (Tregs) (11). CCHE1 has been shown to be functionally correlated with ERK/COX-2 pathway (12). Since COX-2 is expressed in response to inflammatory stimulators and participates in the inflammatory reactions (13), CCHE1 might be involved in the inflammatory responses. Based on the previous reports regarding the role of ANRIL, PICART1, MALAT1, CCAT1, CCAT2, and CCHE1 in the pathogenesis of immune-mediated disorders, we selected these lncRNAs to assess their potential as peripheral markers in the AIDP and CIDP.
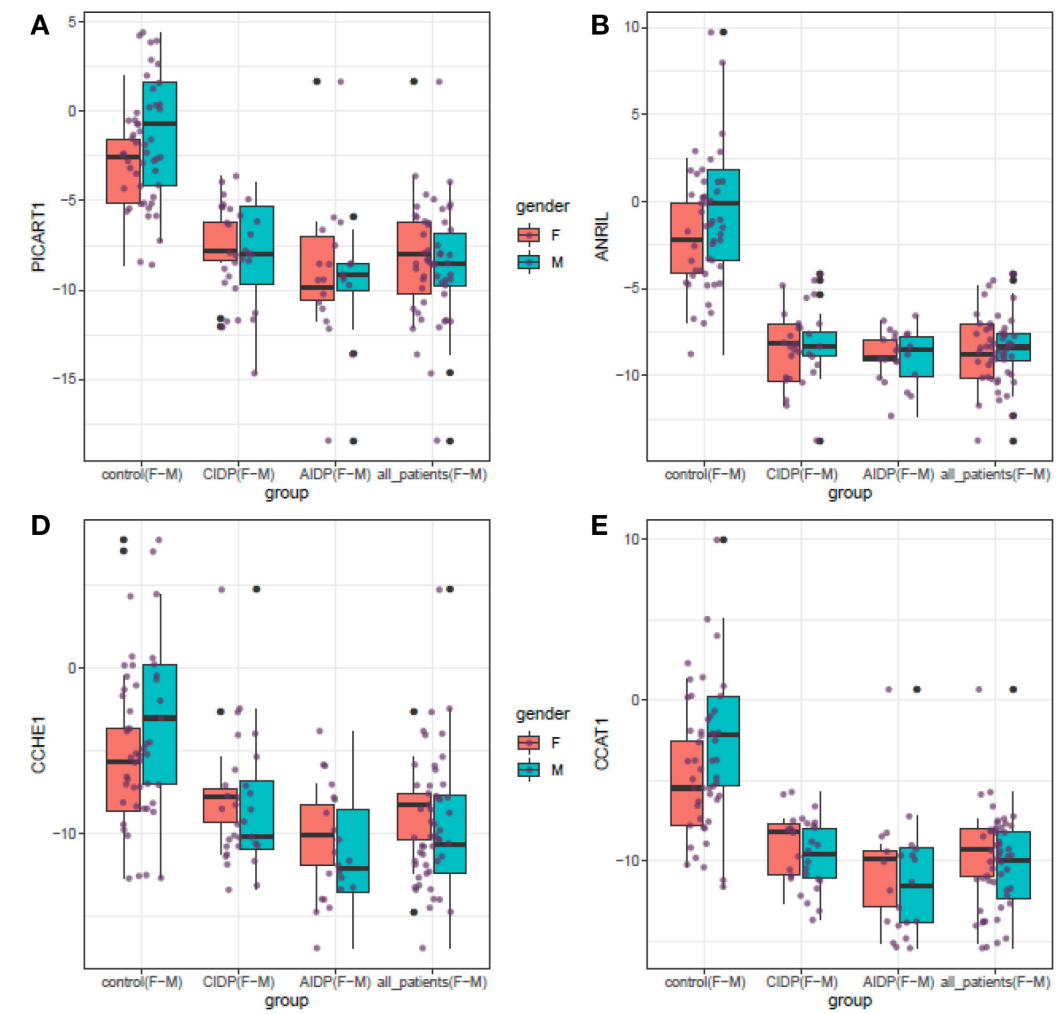
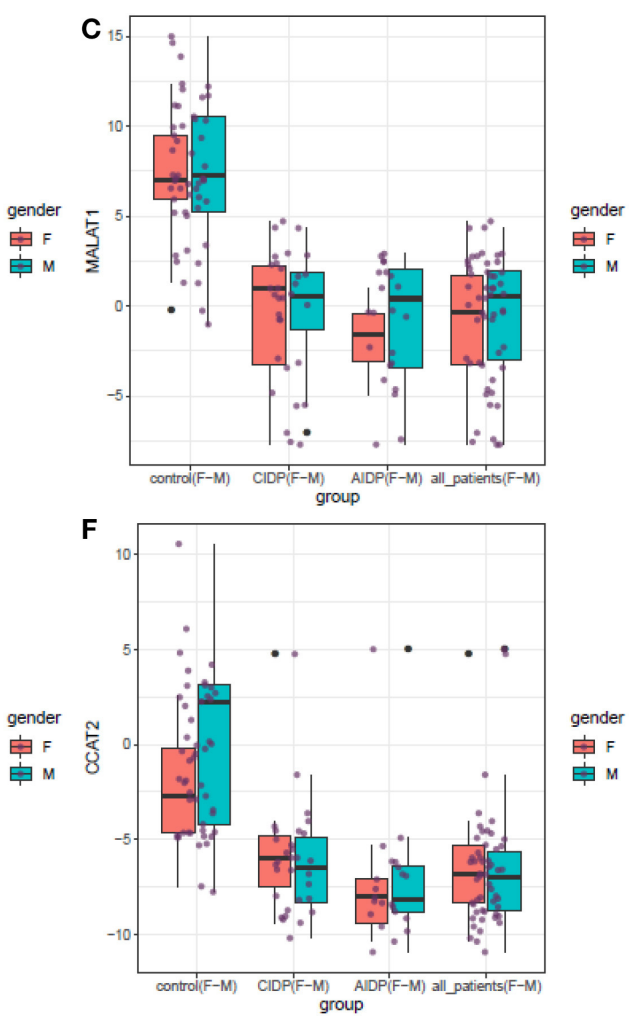

FIGURE 1 | The relative quantification of the expression of ANRIL, PICART1, MALAT1, CCAT1, CCAT2, and CCHE1 IncRNAs in distinct study subgroups including the AIDP, CIDP, and controls. Upper/lower extremes, median, and upper/lower quartiles are shown. Each purple dot represents the gene expression level in one individual. Black dots show outliers (significance level: $p<0.05$ ). 
TABLE 2 | Detailed statistics of the expression analysis of ANRIL, PICART1, MALAT1, CCAT1, CCAT2, and CCHE1 IncRNAs in AIDP, CIDP, and control groups.

\begin{tabular}{|c|c|c|c|c|c|c|c|c|c|c|c|c|c|c|c|c|}
\hline \multirow{2}{*}{\multicolumn{2}{|c|}{ Number of samples }} & \multicolumn{5}{|c|}{ PICART1 } & \multicolumn{5}{|c|}{ ANRIL } & \multicolumn{5}{|c|}{ MALAT1 } \\
\hline & & \multirow{3}{*}{$\begin{array}{c}\text { SE } \\
\\
0.67\end{array}$} & \multirow{3}{*}{$\begin{array}{c}\text { Ratio of mean } \\
\text { expressions } \\
0.02\end{array}$} & \multirow{3}{*}{$\begin{array}{c}P \text {-value } \\
\text { 3.69E-13 }\end{array}$} & \multicolumn{2}{|c|}{$95 \% \mathrm{Cl}$} & \multirow{3}{*}{$\begin{array}{c}\text { SE } \\
\\
0.64\end{array}$} & \multirow{3}{*}{$\begin{array}{c}\begin{array}{c}\text { Ratio of mean } \\
\text { expressions }\end{array} \\
0.01\end{array}$} & \multirow{3}{*}{$\begin{array}{l}P \text {-value } \\
2.89 \mathrm{E}-17\end{array}$} & \multicolumn{2}{|c|}{$95 \% \mathrm{Cl}$} & \multirow{3}{*}{$\begin{array}{l}\text { SE } \\
0.83\end{array}$} & \multirow{3}{*}{$\begin{array}{c}\begin{array}{c}\text { Ratio of mean } \\
\text { expressions }\end{array} \\
0.00\end{array}$} & \multirow{3}{*}{$\begin{array}{c}P \text {-value } \\
8.35 \mathrm{E}-14\end{array}$} & \multicolumn{2}{|c|}{$95 \% \mathrm{Cl}$} \\
\hline \multicolumn{8}{|c|}{ 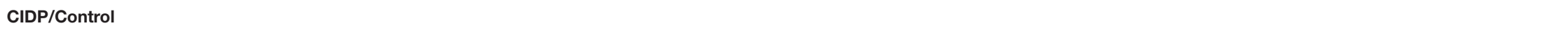 } & & & & & & & & & \\
\hline Total & $31 / 50$ & & & & -7.24 & -4.59 & & & & -8.17 & -5.63 & & & & -9.51 & -6.19 \\
\hline $\mathrm{F}$ & $11 / 25$ & 0.91 & 0.04 & 5.79E-05 & -6.53 & -2.74 & 0.86 & 0.01 & 6.19E-07 & -8.06 & -4.45 & 1.47 & 0.01 & 1.06E-04 & -10.75 & -4.48 \\
\hline M & $20 / 25$ & 0.96 & 0.01 & 3.18E-09 & -9.02 & -5.16 & 0.98 & 0.00 & 3.06E-09 & -9.64 & -5.66 & 1.09 & 0.00 & 2.90E-09 & -10.35 & -5.94 \\
\hline \multicolumn{17}{|c|}{ AIDP/Control } \\
\hline Total & $22 / 50$ & 0.90 & 0.01 & $1.31 \mathrm{E}-09$ & -9.04 & -5.41 & 0.60 & 0.01 & $1.38 \mathrm{E}-19$ & -8.67 & -6.29 & 0.88 & 0.00 & $1.06 \mathrm{E}-12$ & -10.29 & -6.77 \\
\hline $\mathrm{F}$ & $6 / 25$ & 2.10 & 0.04 & $6.77 \mathrm{E}-02$ & -9.95 & 0.48 & 0.68 & 0.01 & $5.18 \mathrm{E}-08$ & -7.81 & -4.93 & 1.11 & 0.00 & $5.23 \mathrm{E}-06$ & -11.33 & -6.45 \\
\hline M & $16 / 25$ & 1.03 & 0.00 & 3.17E-10 & -10.89 & -6.73 & 0.95 & 0.00 & $3.02 \mathrm{E}-10$ & -10.39 & -6.51 & 1.22 & 0.00 & $3.40 E-08$ & -11.10 & -6.13 \\
\hline \multicolumn{17}{|c|}{ CIDP/AIDP } \\
\hline Total & $31 / 22$ & 0.91 & 2.48 & 1.59E-01 & -0.54 & 3.16 & 0.50 & 1.50 & 2.50E-01 & -0.42 & 1.58 & 0.95 & 1.60 & 4.79E-01 & -1.23 & 2.59 \\
\hline $\mathrm{F}$ & $11 / 6$ & 2.17 & 1.07 & 9.64E-01 & -5.13 & 5.33 & 0.86 & 1.08 & 9.01E-01 & -1.73 & 1.95 & 1.59 & 2.43 & 4.33E-01 & -2.11 & 4.66 \\
\hline M & $20 / 16$ & 0.99 & 3.30 & 9.03E-02 & -0.29 & 3.73 & 0.61 & 1.74 & 2.02E-01 & -0.45 & 2.05 & 1.16 & 1.39 & $6.85 \mathrm{E}-01$ & -1.89 & 2.84 \\
\hline \multicolumn{17}{|c|}{ All patients/controls } \\
\hline Total & $53 / 50$ & 0.63 & 0.01 & 2.31E-17 & -7.71 & -5.21 & 0.57 & 0.01 & 5.71E-20 & -8.28 & -6.01 & 0.71 & 0.00 & $6.69 E-20$ & -9.54 & -6.72 \\
\hline F & $17 / 25$ & 0.97 & 0.04 & 4.83E-05 & -6.66 & -2.68 & 0.68 & 0.01 & 2.73E-11 & -7.68 & -4.92 & 1.12 & 0.00 & 3.57E-08 & -10.35 & -5.78 \\
\hline \multirow[t]{2}{*}{ M } & $36 / 25$ & 0.87 & 0.00 & $7.70 \mathrm{E}-12$ & -9.60 & -6.11 & 0.92 & 0.00 & $9.30 \mathrm{E}-10$ & -9.89 & -6.13 & 1.00 & 0.00 & $9.52 \mathrm{E}-11$ & -10.36 & -6.35 \\
\hline & & \multicolumn{5}{|c|}{ CCHE1 } & \multicolumn{5}{|c|}{ CCAT1 } & & & ССАТ2 & & \\
\hline Num & r of samples & SE & $\begin{array}{c}\text { Ratio of mean } \\
\text { expressions }\end{array}$ & $P$-value & & & SE & $\begin{array}{c}\text { Ratio of mean } \\
\text { expressions }\end{array}$ & $P$-value & $95 \%$ & & SE & $\begin{array}{c}\text { Ratio of mean } \\
\text { expressions }\end{array}$ & $P$-value & & $\mathrm{Cl}$ \\
\hline CIDP & ontrol & & & & & & & & & & & & & & & \\
\hline Total & $31 / 50$ & 0.96 & 0.07 & $1.48 \mathrm{E}-04$ & -5.74 & -1.92 & 0.73 & 0.02 & $5.84 \mathrm{E}-11$ & -7.00 & -4.10 & 0.75 & 0.03 & $1.01 \mathrm{E}-09$ & -6.69 & -3.72 \\
\hline $\mathrm{F}$ & $11 / 25$ & 1.26 & 0.22 & $9.27 \mathrm{E}-02$ & -4.76 & 0.38 & 0.86 & 0.06 & 4.32E-05 & -5.85 & -2.35 & 1.26 & 0.11 & $2.45 \mathrm{E}-02$ & -5.88 & -0.47 \\
\hline M & $20 / 25$ & 1.29 & 0.03 & $1.84 \mathrm{E}-04$ & -7.91 & -2.70 & 1.13 & 0.01 & $5.62 \mathrm{E}-07$ & -9.25 & -4.64 & 1.01 & 0.01 & $5.01 E-08$ & -8.99 & -4.88 \\
\hline AIDP/ & ontrol & & & & & & & & & & & & & & & \\
\hline Total & $22 / 50$ & 1.00 & 0.01 & $2.79 \mathrm{E}-08$ & -8.43 & -4.44 & 1.00 & 0.01 & 4.36E-09 & -9.20 & -5.17 & 0.87 & 0.01 & $2.02 \mathrm{E}-09$ & -8.15 & -4.65 \\
\hline $\mathrm{F}$ & $6 / 25$ & 1.56 & 0.04 & $1.04 \mathrm{E}-02$ & -7.97 & -1.27 & 1.24 & 0.02 & $9.62 \mathrm{E}-04$ & -8.83 & -3.19 & 0.92 & 0.02 & $8.92 \mathrm{E}-05$ & -7.80 & -3.72 \\
\hline M & $16 / 25$ & 1.24 & 0.00 & 2.51E-07 & -10.42 & -5.38 & 1.44 & 0.00 & 1.03E-06 & -11.36 & -5.51 & 1.26 & 0.01 & 7.44E-07 & -10.05 & -4.94 \\
\hline CIDP/ & IDP & & & & & & & & & & & & & & & \\
\hline Total & $31 / 22$ & 0.99 & 6.05 & 1.17E-02 & 0.60 & 4.59 & 0.87 & 3.09 & 7.18E-02 & -0.15 & 3.41 & 0.85 & 2.29 & 1.67E-01 & -0.52 & 2.91 \\
\hline $\mathrm{F}$ & $11 / 6$ & 1.39 & 5.40 & 1.13E-01 & -0.70 & 5.57 & 1.22 & 3.77 & $1.54 \mathrm{E}-01$ & -0.89 & 4.72 & 1.38 & 6.00 & 8.11E-02 & -0.36 & 5.53 \\
\hline M & $20 / 16$ & 1.34 & 6.03 & $6.05 \mathrm{E}-02$ & -0.12 & 5.31 & 1.14 & 2.82 & 2.04E-01 & -0.87 & 3.86 & 1.02 & 1.47 & 5.88E-01 & -1.54 & 2.66 \\
\hline All pa & ents/controls & & & & & & & & & & & & & & & \\
\hline Total & $53 / 50$ & 0.86 & 0.03 & $1.30 \mathrm{E}-07$ & -6.62 & -3.21 & 0.74 & 0.01 & $8.95 \mathrm{E}-13$ & -7.71 & -4.75 & 0.68 & 0.02 & $6.54 \mathrm{E}-13$ & -7.06 & -4.34 \\
\hline $\mathrm{F}$ & $17 / 25$ & 1.23 & 0.12 & $1.77 \mathrm{E}-02$ & -5.53 & -0.56 & 0.85 & 0.04 & $1.53 \mathrm{E}-06$ & -6.48 & -3.06 & 0.98 & 0.06 & $2.66 \mathrm{E}-04$ & -6.09 & -2.08 \\
\hline M & $36 / 25$ & 1.10 & 0.01 & 2.95E-07 & -8.66 & -4.26 & 1.15 & 0.01 & 9.04E-08 & -9.94 & -5.27 & 1.01 & 0.01 & 1.75E-08 & -9.22 & -5.14 \\
\hline
\end{tabular}

Bold values mean item with significant difference among two groups. 


\section{MATERIALS AND METHODS}

\section{Enrollment of Patients and Unaffected Controls}

We recruited 22 patients with AIDP, 31 patients with CIDP, and 50 unaffected persons in the present investigation. Unaffected persons recruited as controls had no sign of immune-related conditions. The diagnosis of AIDP and CIDP was based on guidelines stated by the American Academy of Neurology (14) and the National Institute of Neurological Disorders and Stroke (15). Blood samples were taken from patients with AIDP and CIDP in their remission phase. Patients were not on any treatment at the time of sampling. Exclusion criteria were as follows: any recent or chronic infectious process, malignant conditions, and any systemic disorder. The study protocol was approved by the ethical committee of Shahid Beheshti University of Medical Sciences. All enlisted individuals signed the informed consent forms.

\section{Evaluation of the Expression of IncRNAs in Blood Samples}

As the first step, RNA was recovered from blood samples using the commercial RNA extraction Kit (GeneAll, Seoul, South Korea). Then, a fraction of isolated RNA specimens was converted to complementary DNA (cDNA using the OneStep RT-PCR Series Kit (BioFact ${ }^{\mathrm{TM}}$, Seoul, South Korea). Expressions of mentioned lncRNAs were quantified in specimens using the RealQ Plus 2x Master Mix (Amplicon, Denmark). Transcript levels of the B2M gene were quantified and used for
A

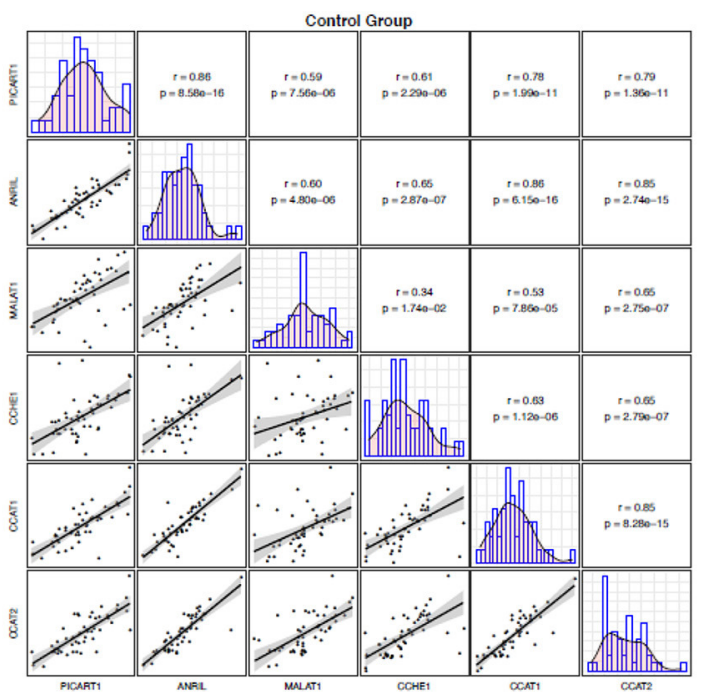

C

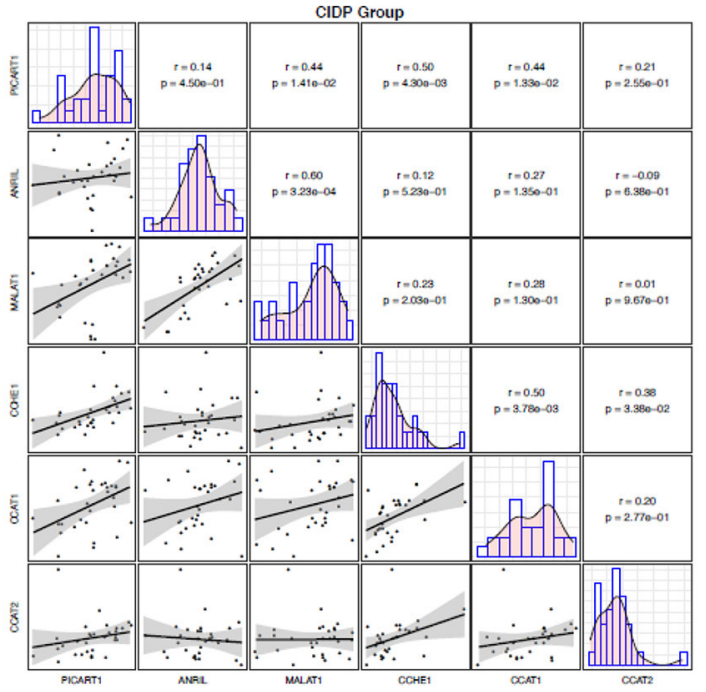

B

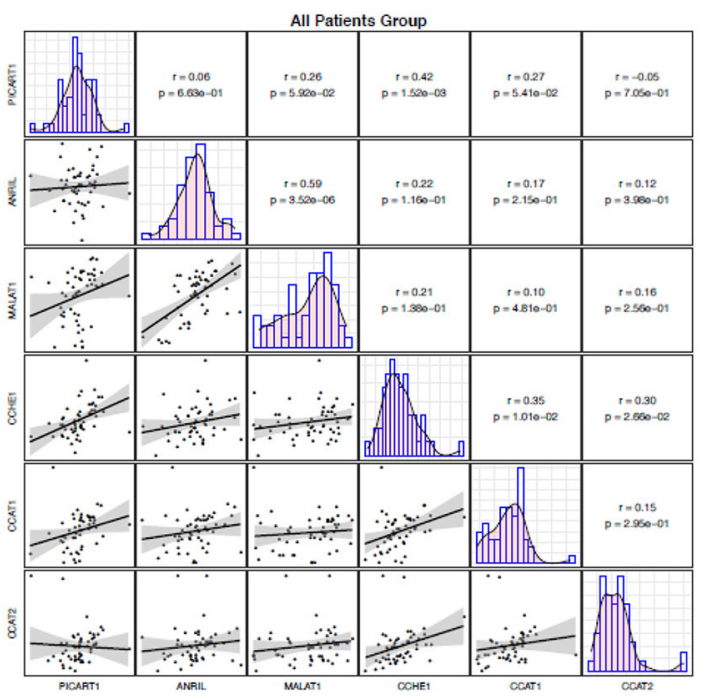

D

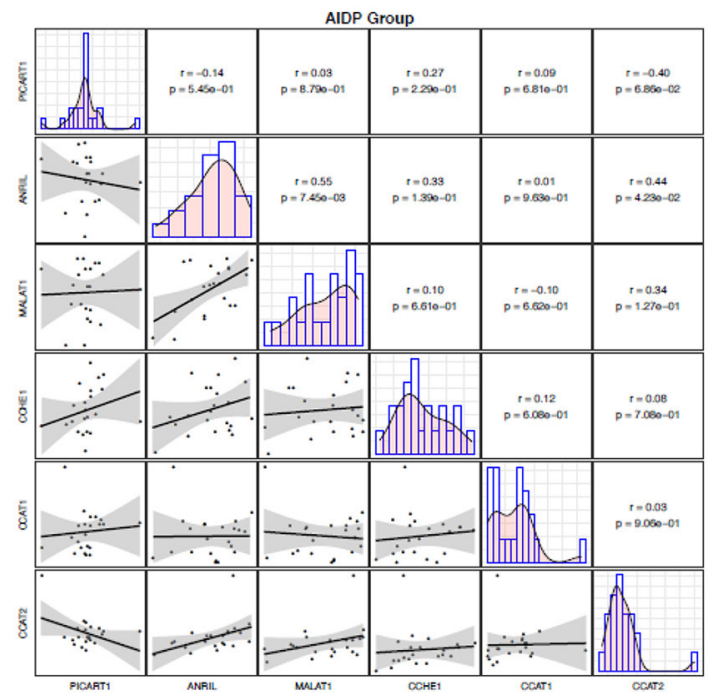

FIGURE 2 | Correlation between the expression levels of ANRIL, PICART1, MALAT1, CCAT1, CCAT2, and CCHE1 IncRNAs in control group (A), all patients (B), CIDP (C), and AIDP (D). The lower panel shows scatter plots and smoothing splines for the expression levels of every possible pair of genes. The upper panel shows the corresponding Pearson's correlation coefficients and $P$ values. 
normalization of transcript levels of lncRNAs. All experiments were executed in duplicate. Primers were designed by Primer3 tool (https://primer3.ut.ee/). The specificity of primers was appraised by in silico tools. PCR products were also run on $2 \%$ agarose gel to verify the presence of expected amplicons and their sizes. Each run had a negative control comprising all PCR reagents except the template. Features of primers and amplified RNA parts are presented in Supplementary Material.

\section{Statistical Methods}

$\mathrm{R}$ program was used for statistical analyses. Transcript quantities of lncRNAs in relation to the B2M gene were quantified

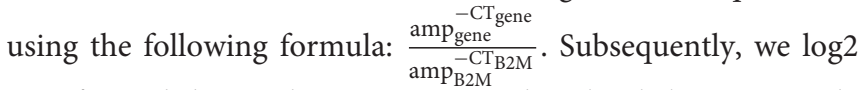
transformed these values to compare their levels between study subgroups. The mean values of gene expression levels were compared between subgroups using the $t$-test. Correlations between RNA levels were judged using Spearman's correlation coefficients. Receiver operating characteristic (ROC) curves were depicted to appraise the diagnostic power of lncRNAs. At first,
Bayesian generalized linear model, generalized linear model, and linear discriminant analysis with 10 -fold cross validation were used to measure the related parameters. Then, the best model was used for further estimations.

\section{RESULTS}

\section{Global Information About Enlisted Individuals}

Global data of enlisted persons in the AIDP, CIDP, and control groups are denoted in Table 1.

\section{Expression Analyses}

Figure 1 displays the relative quantification of ANRIL, PICART1, MALAT1, CCAT1, CCAT2, and CCHE1 lncRNAs levels in distinct study subgroups including the AIDP, CIDP, and controls.

ANRIL, PICART1, MALAT1, CCAT1, CCAT2, and CCHE1 lncRNAs were significantly downregulated in both cases with AIDP and CIDP compared with unaffected individuals. Genderbased comparisons also verified such downregulations in both
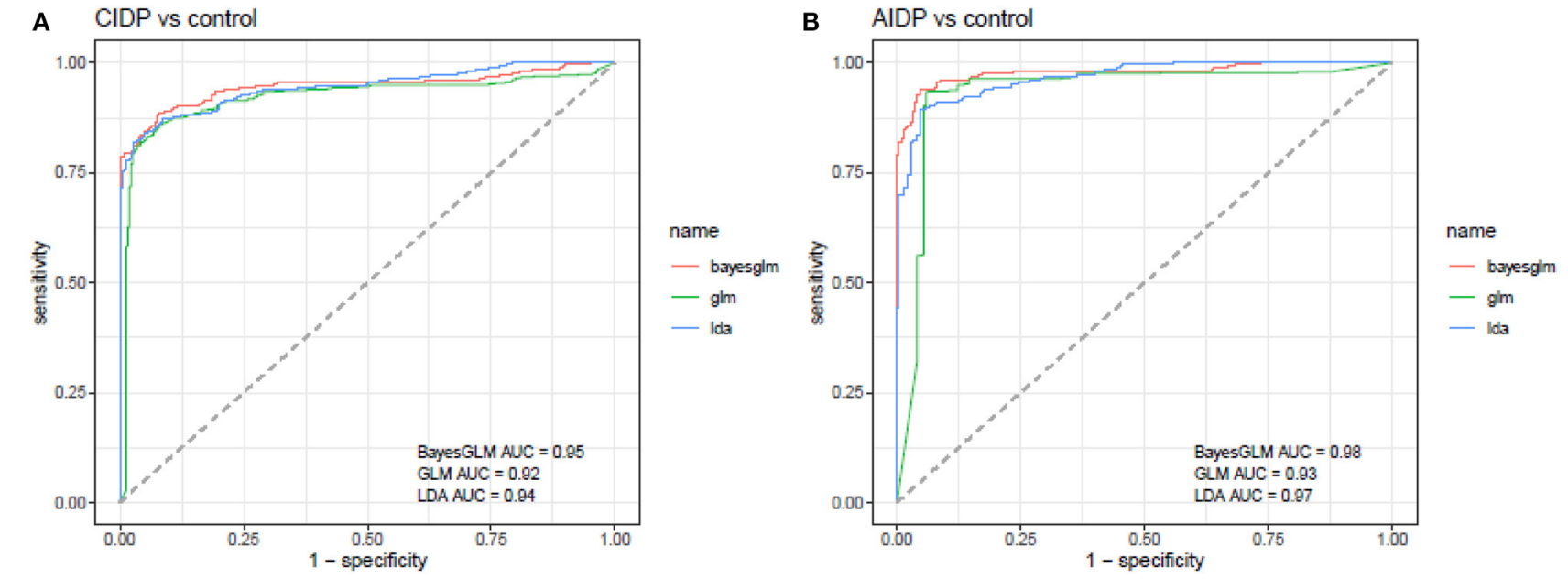

\section{CIDP vs AIDP}

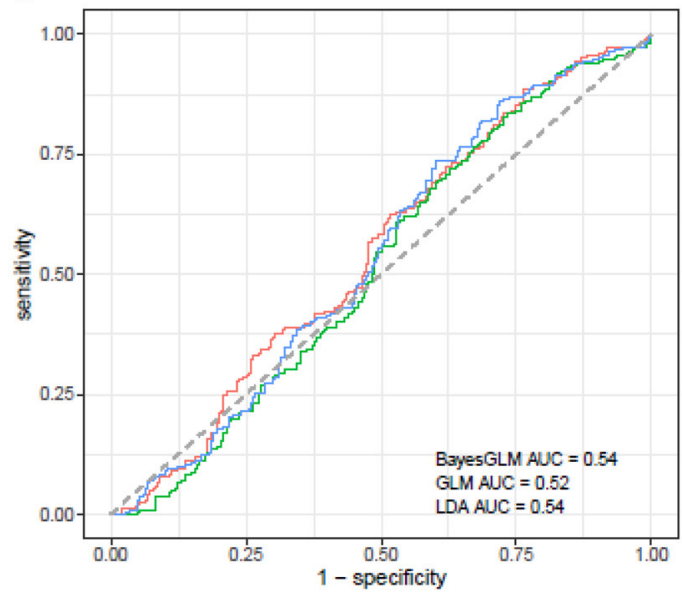

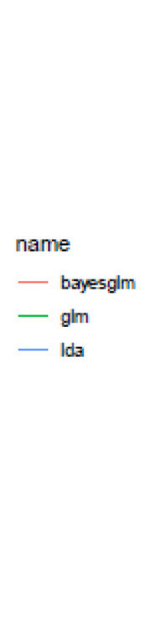

D

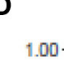

1.00

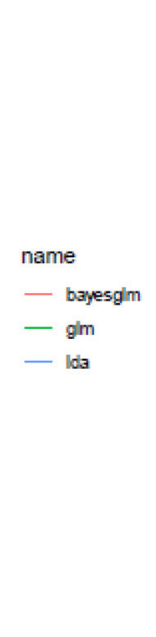

FIGURE 3 | ROC curves depicted using Bayesian generalized linear model, generalized linear model, and linear discriminant analysis (AUC, area under curve). 


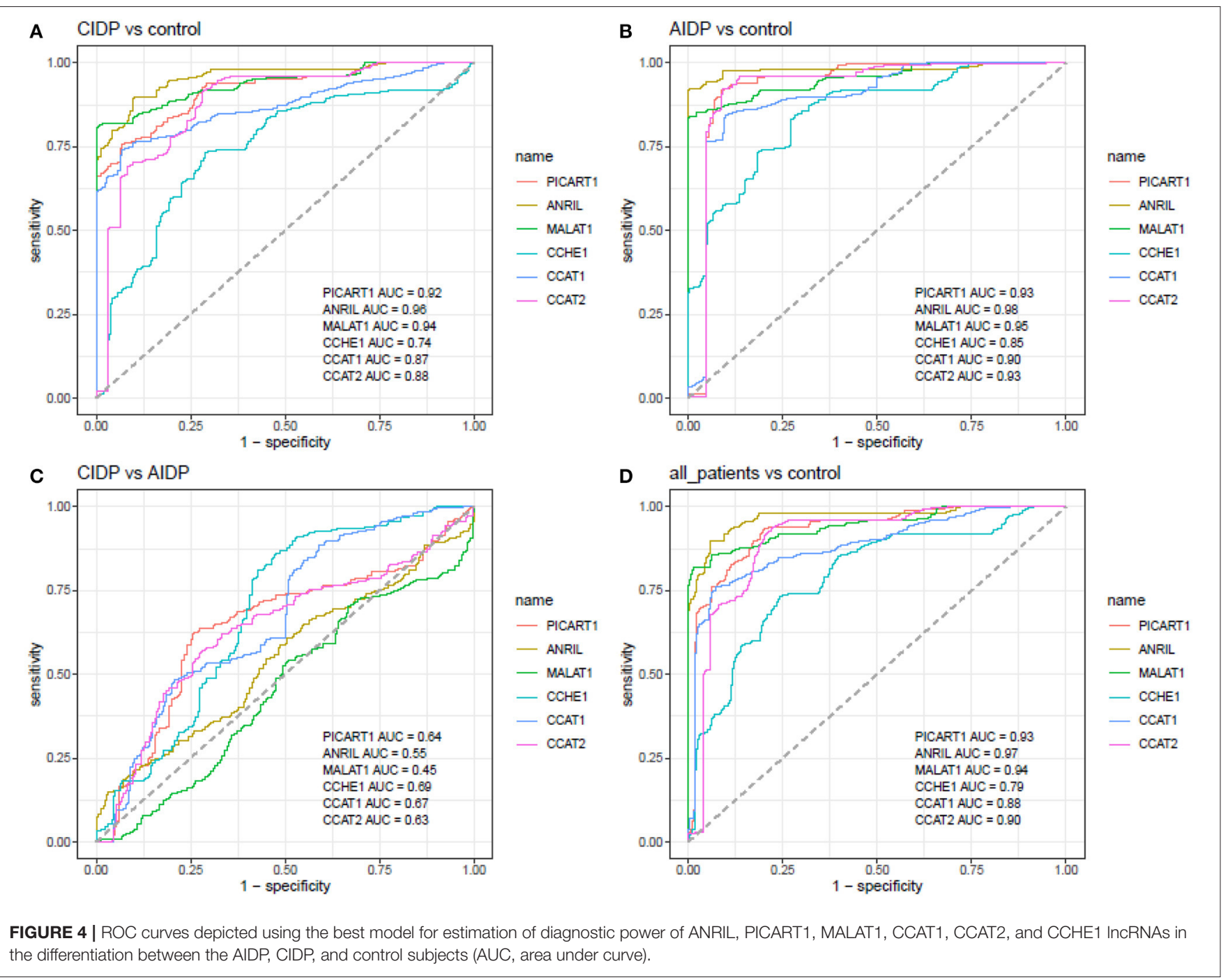

male and female subjects compared with sex-matched unaffected controls for all lncRNAs. There was no significant difference in the expression of either lncRNA between cases with AIDP and cases with CIDP. Table 2 demonstrates the detailed parameters obtained from statistical analyses.

While the expression levels of ANRIL and PICART1 were significantly correlated in healthy subjects $(r=0.86, p=8.5 \mathrm{E}$ 16), similar analysis in cases with AIDP and CIDP revealed no significant correlation. Among healthy subjects, correlation coefficients ranged from 0.86 between ANRIL and PICART1 to 0.34 between MALAT1 and CCHE1. In patients, the most robust correlation among patients was detected between ANRIL and MALAT1 lncRNAs ( $\mathrm{r}=0.59, p=3.52 \mathrm{E}-6)$, while the minimum correlation coefficient was detected between ANRIL and PICART1 $(r=0.06, p=6.63 \mathrm{e}-1)$. In cases with CIDP, correlation coefficients ranged from 0.01 (MALAT1/CCAT2) to 0.50 (PICART1/CCHE1 and CCAT2/CCHE1). Finally, among cases with AIDP, correlation coefficients ranged from 0.01 (ANRIL/CCAT2) to 0.55 (ANRIL/MALAT1). Figure 2 demonstrates correlation coefficients between
lncRNAs pairs and significance levels in distinct study subgroups.

\section{Receiver Operating Characteristic Curves}

Receiver operating characteristic curves were depicted using Bayesian generalized linear model, generalized linear model, and linear discriminant analysis (Figure 3). Then, the best model was used for further estimations (Figure 4).

ANRIL, MALAT1, and PICART1 had the diagnostic power of $0.96,0.94$, and 0.92 in distinguishing between cases with CIDP and controls, respectively. A combination of all lncRNAs resulted in 0.95 diagnostic power with a sensitivity of 0.85 and specificity of 0.96 for this purpose. Diagnostic power values of these IncRNAs in the differentiation between cases with AIDP and controls were 0.98, 0.95, and 0.93, respectively. The combinatorial diagnostic power reached 0.98 for the differentiation between cases with AIDP and controls. The sixlncRNA panel could differentiate combined cases with AIDP and CIDP from controls with AUC, sensitivity, and specificity values of 0.97, 0.90, and 0.96, respectively (Table 3). 


\section{DISCUSSION}

Based on the importance of finding biomarkers for immunemediated neuropathies, we investigated the expression patterns of a panel of lncRNAs in patients with acute and chronic forms of this condition. Notably, we detected remarkable downregulation of ANRIL, PICART1, MALAT1, CCAT1, CCAT2, and CCHE1 in cases with both AIDP and CIDP compared with unaffected individuals. The significance of downregulation of some of these lncRNAs in the modulation of immune responses has been verified in other disorders. For instance, PICART1 suppression has been shown to result in JAK2/STAT3 activation (6). STAT3 has a critical role in the regulation of innate and adaptive immune reactions via modulating the expression of numerous cytokines such as interferons and interleukins-2,-6,-10, and-12 (16). The over-activation of STAT3 has a prominent role in the induction of autoimmune reactions in a wide range of tissues (17). Therefore, downregulation of PICART1 might participate in the pathogenesis of immune-mediated neuropathies through the activation of STAT3. Moreover, PICART1 interacts with AKT/GSK3 $\beta / \beta$-catenin signaling pathway (18). Notably, GSK3 and $\beta$-catenin regulate the innate immune response against RNA and DNA viruses (19). Based on the importance of viral infections in the initiation of AIDP (20), the interplay between PICART1 and these two proteins might be implicated in the pathogenesis of this form of immune-related neuropathies. MALAT1 has been shown to induce the expansion of tolerogenic DCs and Tregs through the miR155/DC-SIGN/IL-10 axis (21). Both mentioned cell populations contribute to the modulation of immune responses during the course of immune-related neuropathies $(22,23)$. Thus, this axis might also mediate the role of MALAT1 in the pathobiology of these conditions. CCAT1 has been shown to interact with miR-375-3p to decrease the expression of IRF5 (24), a transcription factor that controls the expression of several genes participating in the inflammatory reactions and induction of the immune responses (25). Therefore, the downregulation of CCAT1 might influence the pathogenesis of AIDP or CIDP via this route. The expression of CCHE1 has been correlated with the COX-2 pathway (12). The expression of COX-2 by macrophages might participate in the production of prostaglandins during the acute phase of demyelination in immune-related neuropathies (26). Therefore, the downregulation of CCHE1 in patients with immunerelated neuropathies might be a compensative mechanism for amelioration of COX-2-related demyelinating effects in the acute phase. This speculation is supported by the fact that recruited patients in the current study were in the remission phase.

Although the functional consequences of the ANRIL downregulation have not been evaluated in the context of immune-related disorders, decreased levels of this lncRNA have also been noted in the peripheral blood of patients with periodontitis, a condition which is caused by the interplay between immune reactions and pathogens (27). The expression of ANRIL has been shown to be regulated by TNF- $\alpha$ (5), a cytokine with dual roles in the pathogenesis of immune-related neuropathies (28). Therefore, the functional relevance of the ANRIL downregulation should be appraised in these conditions. 
Gender-based comparisons also verified the observed downregulations of lncRNAs in both male and female subjects compared with sex-matched unaffected controls. Therefore, the contribution of these lncRNAs in the pathogenesis of immunemediated neuropathies is sex-independent. Moreover, there was no significant difference in the expression of either lncRNA between cases with AIDP and cases with CIDP, suggesting shared molecular mechanisms for these conditions in the terms of participation of mentioned lncRNAs.

The assessment of pairwise correlations between lncRNAs revealed distinct patterns among patients and healthy subjects. For instance, while the expression levels of ANRIL and PICART1 were significantly correlated in the healthy subjects, similar analysis in the cases with AIDP and CIDP revealed no significant correlation. The most robust correlation among patients was detected between ANRIL and MALAT1 lncRNAs. These findings indicate the influence of the disease condition on the correlation network between lncRNAs. Moreover, the altered interactions between lncRNAs might be involved in the pathogenesis of immune-related neuropathies.

The most important finding of our study has been the robust ability of the mentioned lncRNAs in distinguishing between patients with immune-related neuropathies and healthy status. The best values have been obtained for ANRIL, MALAT1, and PICART1. A combination of all lncRNAs resulted in 0.95 diagnostic power with a sensitivity of 0.85 and specificity of 0.96 for this purpose. These values are superior to presently used markers in this disorder. Most notably, the six-lncRNA panel could differentiate cases from controls with AUC, sensitivity, and specificity values of $0.97,0.90$, and 0.96, respectively. Therefore, we suggest this lncRNA panel as a

\section{REFERENCES}

1. Kieseier BC, Mathey EK, Sommer C, Hartung H-P. Immunemediated neuropathies. Nat Rev Dis Primers. (2018) 4:123. doi: 10.1038/s41572-018-0027-2

2. Brannagan III TH. Current diagnosis of CIDP: the need for biomarkers. J Peripheral Nervous Syst. (2011) 16:313. doi: 10.1111/j.1529-8027.2011.00298.x

3. Wang J, Wei F, Zhou H. Advances of lncRNA in autoimmune diseases. Front Lab Med. (2018) 2:79-82. doi: 10.1016/j.flm.2018.07.004

4. Hur K, Kim S-H, Kim J-M. Potential implications of long noncoding RNAs in autoimmune diseases. Immune Netw. (2019) 19:e4. doi: 10.4110/in.2019.19.e4

5. Zhou X, Han X, Wittfeldt A, Sun J, Liu C, Wang X, et al. Long non-coding RNA ANRIL regulates inflammatory responses as a novel component of NF-кB pathway. RNA Biol. (2016) 13:98-108. doi: 10.1080/15476286.2015.1122164

6. Zhao JM, Cheng W, He XG, Liu YL, Wang FF, Gao YF. Long non-coding RNA PICART1 suppresses proliferation and promotes apoptosis in lung cancer cells by inhibiting JAK2/STAT3 signaling. Neoplasma. (2018) 65:77989. doi: 10.4149/neo_2018_171130N778

7. Ciofani M, Madar A, Galan C, Sellars M, Mace K, Pauli F, et al. A validated regulatory network for Th17 cell specification. Cell. (2012) 151:289303. doi: 10.1016/j.cell.2012.09.016

8. Li Z, Zhang Q, Wu Y, Hu F, Gu L, Chen T, et al. lncRNA Malatl modulates the maturation process, cytokine secretion and apoptosis in airway sensitive and specific diagnostic panel for acquired immunemediated polyneuropathies.

\section{DATA AVAILABILITY STATEMENT}

The raw data supporting the conclusions of this article will be made available by the authors, without undue reservation.

\section{ETHICS STATEMENT}

The studies involving human participants were reviewed and approved by IR.SBMU.MSP.REC.1398.849. The patients/participants provided their written informed consent to participate in this study.

\section{AUTHOR CONTRIBUTIONS}

$\mathrm{BH}$ and $\mathrm{FN}$ performed the experiment. AS and $\mathrm{HH}$ analyzed the data. SG-F and MT wrote the draft and revised it. All authors approved the submitted version.

\section{FUNDING}

This study was financially supported (Grant Number 21974) from the Shahid Beheshti University of Medical Sciences.

\section{SUPPLEMENTARY MATERIAL}

The Supplementary Material for this article can be found online at: https://www.frontiersin.org/articles/10.3389/fimmu. 2021.643615/full\#supplementary-material

epithelial cell-conditioned dendritic cells. Exp Ther Med. (2018) 16:39518. doi: 10.3892/etm.2018.6687

9. Liu J, Ding D, Jiang Z, Du T, Liu J, Kong Z. Long non-coding RNA CCAT1/miR-148a/PKC $\zeta$ prevents cell migration of prostate cancer by altering macrophage polarization. Prostate. (2019) 79:105-12. doi: 10.1002/pros.23716

10. Wu Z, Li Y, Wu Y, Wang Y, Nian W, Wang L, et al. Long non-coding RNA CCAT2 promotes the breast cancer growth and metastasis by regulating TGF-beta signaling pathway. Eur Rev Med Pharmacol Sci. (2017) 21:706-14.

11. Sanjabi S, Oh SA, Li MO. Regulation of the immune response by TGF- $\beta$ : from conception to autoimmunity and infection. Cold Spring Harbor Perspect Biol. (2017) 9:a022236. doi: 10.1101/cshperspect.a022236

12. Gaballah HH, Gaber RA, Elrashidy MA, Elshahat DA, Hablus MA, Ebeid AM. Expression of long non-coding RNA CCHE1 in colorectal carcinoma: correlations with clinicopathological features and ERK/COX-2 pathway. $\mathrm{Mol}$ Biol Rep. (2019) 46:657-67. doi: 10.1007/s11033-018-4521-0

13. Simon LS. Role and regulation of cyclooxygenase-2 during inflammation. Am J Med. (1999) 106:37S-42S. doi: 10.1016/S0002-9343(99) 00115-1

14. Neurology AAo. Research criteria for diagnosis of chronic inflammatory demyelinating polyneuropathy (CIDP): report from an ad hoc subcommittee of the American Academy of Neurology AIDS Task Force. Neurology. (1991) 41:617-8. doi: 10.1212/WNL.41.5.617

15. Van der Meché F, Van Doorn P, Meulstee J, Jennekens F. Diagnostic and classification criteria for the Guillain-Barré syndrome. Eur Neurol. (2001) 45:133-9. doi: 10.1159/000052111 
16. Harris TJ, Grosso JF, Yen H-R, Xin H, Kortylewski M, Albesiano E, et al. Cutting edge: an in vivo requirement for STAT3 signaling in TH17 development and TH17-dependent autoimmunity. J Immunol. (2007) 179:4313-7. doi: 10.4049/jimmunol.179.7.4313

17. Flanagan SE, Haapaniemi E, Russell MA, Caswell R, Allen HL, De Franco E, et al. Activating germline mutations in STAT3 cause early-onset multi-organ autoimmune disease. Nat Genet. (2014) 46:812-4. doi: 10.1038/ng.3040

18. Cao Y, Lin M, Bu Y, Ling H, He Y, Huang C, et al. p53-inducible long noncoding RNA PICART1 mediates cancer cell proliferation and migration. Int J Oncol. (2017) 50:1671-82. doi: 10.3892/ijo.2017.3918

19. Marineau A, Khan KA, Servant MJ. Roles of GSK-3 and $\beta$-catenin in antiviral innate immune sensing of nucleic acids. Cells. (2020) 9:897. doi: 10.3390/cells9040897

20. Hadden RD, Karch H, Hartung HP, Zielasek J, Weissbrich B, Schubert J, et al. Preceding infections, immune factors, and outcome in Guillain-Barré syndrome. Neurology. (2001) 56:758-65. doi: 10.1212/WNL.56.6.758

21. Wu J, Zhang $\mathrm{H}$, Zheng Y, Jin X, Liu M, Li S, et al. The long noncoding RNA MALAT1 induces tolerogenic dendritic cells and regulatory $\mathrm{T}$ cells via miR155/dendritic cell-specific intercellular adhesion molecule-3 grabbing nonintegrin/IL10 axis. Front Immunol. (2018) 9:1847. doi: 10.3389/fimmu.2018.01847

22. Quan S, Kim H-J, Dukala D, Sheng JR, Soliven B. Impaired dendritic cell function in a spontaneous autoimmune polyneuropathy. J Immunol. (2015) 194:4175-84. doi: 10.4049/jimmunol.1401766

23. Wolbert J, Cheng MI, Meyer zu Horste G, Su MA. Deciphering immune mechanisms in chronic inflammatory demyelinating polyneuropathies. JCI Insight. (2020) 5:e132411. doi: 10.1172/jci.insight.132411

24. Liu Z, Ma C, Tang X, Tang Q, Lou L, Yu Y, et al. The reciprocal interaction between IncRNA CCAT1 and miR-375-3p contribute to the downregulation of IRF5 gene expression by solasonine in HepG2 human hepatocellular carcinoma cells. Front Oncol. (2019) 9:1081. doi: 10.3389/fonc.2019. 01081

25. Kaur A, Lee L-H, Chow S-C, Fang C-M. IRF5-mediated immune responses and its implications in immunological disorders. Int Rev Immunol. (2018) 37:229-48. doi: 10.1080/08830185.2018. 1469629

26. Hu W, Mathey E, Hartung HP, Kieseier BC. Cyclo-oxygenases and prostaglandins in acute inflammatory demyelination of the peripheral nerve. Neurology. (2003) 61:1774-9. doi: 10.1212/01.WNL.0000098884.75756.4D

27. Gholami L, Ghafouri-Fard S, Mirzajani S, Arsang-Jang S, Taheri M, Dehbani Z, et al. The lncRNA ANRIL is down-regulated in peripheral blood of patients with periodontitis. Noncoding RNA Res. (2020) 5:606. doi: 10.1016/j.ncrna.2020.04.001

28. Wang Y, Zhang J, Luo P, Zhu J, Feng J, Zhang HL. Tumor necrosis factor- $\alpha$ in Guillain-Barré syndrome, friend or foe? Expert Opin Ther Targets. (2017) 21:103-12. doi: 10.1080/14728222.2017.1258402

Conflict of Interest: The authors declare that the research was conducted in the absence of any commercial or financial relationships that could be construed as a potential conflict of interest.

Copyright (c) 2021 Hussen, Nicknafs, Hidayat, Sayad, Ghafouri-Fard and Taheri. This is an open-access article distributed under the terms of the Creative Commons Attribution License (CC BY). The use, distribution or reproduction in other forums is permitted, provided the original author(s) and the copyright owner(s) are credited and that the original publication in this journal is cited, in accordance with accepted academic practice. No use, distribution or reproduction is permitted which does not comply with these terms. 